\title{
Joint Space-Time Block-Coding and Beamforming for the Multi-user Radio Over Plastic Fiber Downlink
}

\author{
Yichuan Li* Student Member, IEEE, Mohammed El-Hajjar* Senior Member, IEEE, Lajos Hanzo* Fellow, IEEE
}

${ }^{*}$ School of ECS, University of Southampton, UK

\begin{abstract}
An all-optical processing aided multi-functional wireless Multiple-Input-Multiple-Output (MIMO) architecture is designed, where both diversity and beamforming gains can be attained. Explicitly, we propose an architecture, where the twin-antenna Alamouti space-time block coded symbols are transmitted using a single Mach-Zenhder Modulator (MZM) over a plastic optical fiber. The signal is then transmitted through a set of Fiber Bragg Gratings (FBGs) for attaining the appropriate phase shifts for beamforming, followed by transmission over the wireless channel. The attainable angular beamsteering range is about $150^{\circ}$, where beamsteering is realized without the need for a large number of complex electronic phase shifter networks. The tuning of the beamsteering angle can be readily implemented by carefully controlling the drive voltage in the Mach-Zenhder Modulator, where we use MZM aided multi-wavelength generation for supporting a large number of antennas.
\end{abstract}

Index terms Beamforming, Plastic Optical Fiber, Multifunctional MIMO, Mach-Zenhder Modulator.

\section{Motivation And Challenges}

Optical fiber aided wireless beamforming is attractive compared to its electronic phase shifter assisted counterpart, especially once fiber in the home (FITH) relying on lowcost plastic optical fiber becomes a reality [1], [2]. Plastic Optical Fiber (POF) is regarded as a strong candidate for future indoor communication systems, since it provides a lowcost solution to indoor communications due to its convenient installation [1]. However, POF techniques are in their infancy, like silicon was decades ago, which motivated our research. When reaching maturity, POF may even replace the plastic insulation of mains-wiring. On a similar note, multifunctional Multiple-Input-Multiple-Output (MIMO) systems constitute promising techniques, since both diversity as well as multiplexing and beamforming gains can be attained in order to reduce the effect of multipath fading and to increase both the channel capacity and Signal-to-Noise Ratio (SNR) gains [3], [4]. Furthermore, the standardization of different MIMO techniques including transmitter beamforming in the IEEE 802.11 family used for Wi-Fi [5] motivates the implementation of the MIMO techniques in the indoor environment. Hence, we aim for designing an all-optical processing aided multifunctional MIMO (MF-MIMO) assisted indoor system, which

Copyright (c) 2015 IEEE. Personal use of this material is permitted However, permission to use this material for any other purposes must be obtained from the IEEE by sending a request to pubs-permissions@ieee.org

The financial support of the EPSRC projects EP/Noo4558/1 and EP/L018659/1, as well as of the European Research Council's Advanced Fellow Grant under the Beam-Me-Up project and of the Royal Society's Wolfson Research Merit Award is gratefully acknowledged.

The authors are with the School of Electronics and Computer Science, University of Southampton, Southampton SO17 1BJ, U.K. (e-mail: yl2e13@ ecs.soton.ac.uk; meh@ecs.soton.ac.uk; 1h@ecs.soton.ac.uk). achieves both diversity as well as beamforming gains and supports multiple users relying on all-optical processing by the POF, while minimising the number of electronics components and processing cost [6].

A number of challenges are imposed by the current MFMIMO aided indoor networking techniques, which can be summarised as follows:

1) State-of-the-art beamforming techniques mainly rely on invoking phase shifters in the transceiver, which impose a high insertion loss [2].

2) Numerous phase shifters are needed for beamforming in large-scale MF-MIMO systems, such as Layered Steered Space-Time Codes (LSSTC) and Layered Steered Space-Time Spreading (LSSTS) [4], [7].

3) Since each MZM can only modulate a single RF signal, hence multiple optical modulators are required for transmitter diversity or multiplexing techniques, such as Space Time Block Coding (STBC) [8] and their number is proportional to the number of transmitters, which potentially results in high complexity [9].

The first challenge can be tackled by using fiber optical solutions, such as a chirped fiber brag grating (CFBG) [10], relying on the wavelength-dependent time delay imposed by CFBG. Therefore, the complexity of the MF-MIMO configuration can be substantially reduced by avoiding bulky phase shifters [10]. The optical solution is capable of reducing the loss imposed by an electronic phase shifter and significantly simplifies the wireless beamforming [2].

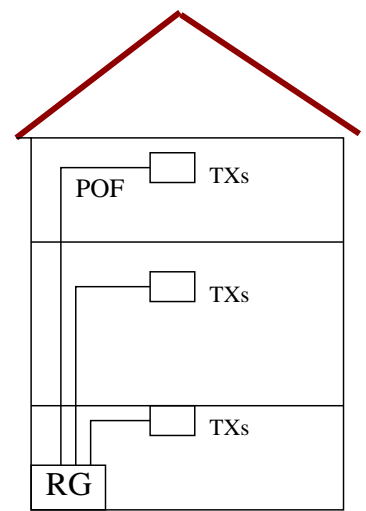

Figure 1: A FITH Architecture based on POF. TXs: Transmitters, RG: Residential Gateway, POF: Plastic Optical Fiber

As for the third challenge, a single optical double-sideband (ODSB) modulation solution was proposed in [11] for simplifying the system by encoding the related pair of signals into two different side bands of an ODSB signal using optical 
up-conversion. However, no beamforming can be realized using the architecture of [11], unless we expand the same architecture to an increased number of antenna elements.

Therefore, we propose the all-optical processing aided downlink architecture of Figure 1 conceived for an indoor environment, which amalgamates both STBC and beamforming by exploiting the nonlinearity of the Mach-Zenhder Modulator (MZM) and of the CFBG in order to address all three of the above mentioned challenges. We propose STBC as an example of a transmitter diversity scheme, noting that our system is quite flexible and hence it is also readily applicable to other sophisticated MIMO techniques, such as V-BLAST [3], Spatial Modulation or Space-time Shift Keying (STSK) [12]. Combining STBC and beamforming has been proposed in the literature [3], [4], [7], where it was shown that the combination of these two techniques attains a better bit-errorrate performance than each of the individual techniques [3], [4], [7]. Additionally, based on the capacity analysis presented in [13], in non-line-of-sight channels, the combination of the two techniques provides a higher capacity than each individual technique. The beamforming employed in this paper is analog beamforming, which requires the knowledge of the Angle of Departure (AoD) at the transmitter. The AoD can be estimated using the techniques reported in [14], [15]. On the other hand, STBC requires the knowledge of the channel impulse response at the receivers, which can be estimated as reported in [16].

In order to generate the twin-antenna aided STBC symbols [8] whilst relying on a single wavelength, we will use a combination of two Laser Diodes (LDs) and a pair of optical band-pass filters. Their output signal will be fed into a MZM. Then the MZM copies the STBC signals to multiple wavelengths by exploiting the nonlinearity of MZM [17]. The wavelengths can be tuned by a frequency-controllable MZM drive signal in order to control the beamsteering angle. These signal processing operations will be carried out by the residential gateway (RG) of Figure 1 (the link between the access and the in-home network). Afterwards, a POF is invoked for supporting a multi-user system in each floor of Figure 1 by relying on the mode division multiplexing principle [18], where each mode is capable of carrying the STBC symbols of a different user. Then, by exploiting the time delay of CFBG and by invoking the appropriate photodetection and demodulation, a beamforming angular range of approximately $150^{\circ}$ can be achieved for each transmitted signal corresponding to each STBC symbol.

The novelty of this paper can be summarised as follows:

1) The MF-MIMO principle can be readily supported by optical fiber based processing without requiring a large number of phase shifters and multiple optical modulators, hence substantially reducing the complexity of wireless beamforming systems additionally supporting the STBC.

2) We design a novel tunable microwave generator based on the MZM's nonlinearity for controlling the beamforming pattern, whilst also providing a less costly alternative in comparison to the state-of-the-art commercial microwave generators.

3) By conveniently controlling the number of side-bands with the aid of the MZM's nonlinearity, the system becomes quite scalable and it is capable of supporting larger antenna arrays for the sake of achieving narrower beams.

4) Mode division multiplexing (MDM) is implemented within a plastic optical fiber for supporting multiple users, where the multiple STBC signals of different users can be readily mapped to different modes.

The rest of the paper is organised as follows. In Section II, the proposed system architecture will be presented, while our performance results are discussed in Section III, followed by our conclusions in Section IV.

\section{Proposed System Model}

In this paper, we propose a multi-user system for the downlink of an in-home network, where each user's signal is encoded as a $2 \times 1$ STBC signal modulated onto a doubleside-band optical carrier and transmitted over plastic optical fiber. Each signal of the STBC can be beamsteered by using all-optical processing. Thus, the system of Figure 2 can be expanded to support multi-user applications by simply copying the same design and transmitting the different users' signals in different modes of the POF using MDM, which is shown in Figure 3. To further detail the rationale of our proposed system, we will simply describe a single-user model and characterise the spectrum of the signal at each processing stage in the network.

As shown in Figure 2, a modulated optical double sideband (ODSB) signal is generated by exploiting two directmodulated laser diodes (LDs), with each bandlimited by an Optical Band Pass Filter (OBPF). Using the OBPFs of Figure 2 , the upper side band of the upper LD and the lower side band of the lower LD are removed, while the remaining side-bands are combined. Then, the combined signal, which contains the data streams $x_{1}$ and $x_{2}$ in the separate side-bands is fed into the MZM2 of Figure 2. Here, the pair of data streams $x_{1}$ and $x_{2}$ correspond to the pair of symbols transmitted from the two antennas of the Alamouti Scheme, with $x_{1}$ fed into Transmitter Antenna Array 1 (TAA1) and $x_{2}$ fed into Transmitter Antenna Array 2 (TAA2) as shown in Figure 2 [8].

In our design, we generate a multi-wavelength signal for supporting beamforming as motivated below. In order to realize directional beamsteering, when employing linear uniform arrays, each element requires differently delayed signals, where the time delay difference between the neighbouring elements must be constant. Since CFBG imposes a linear time delay on the carrier, it can be invoked for the beamforming scheme by exploiting the time delay difference of the differentwavelength carriers in CFBG. Thus, a multi-wavelength signal carrying the same signal at each wavelength having a constant wavelength-spacing is required, whilst guaranteeing a constant time delay difference between neighbouring elements.

Here, the higher order side-bands introduced by the nonlinearity of MZM2 seen in Figure 2 can generate a multiwavelength signal, and each wavelength carries a set of STBC signal. The STBC signal is transmitted as ODSB and copied to different wavelengths. Then, the multi-wavelength signal generated for each user is transmitted in a single mode of the POF, while the different users' signals are transmitted in different modes. The CFBG of Figure 2 seen at the output of the POF provides the different wavelengths of the multi-wavelength signal associated with different delays, thus 


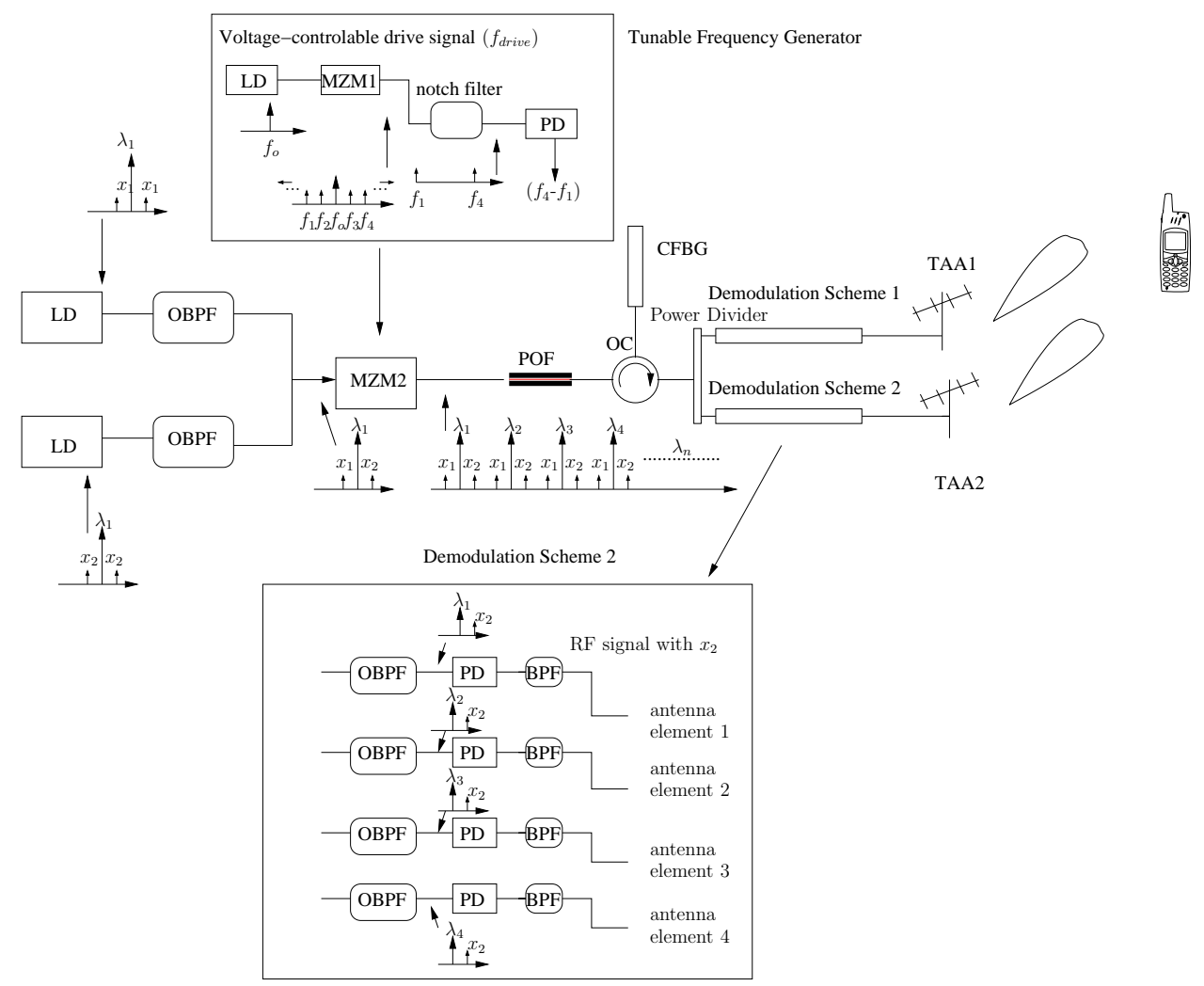

Figure 2: Proposed System Model based on a Single User

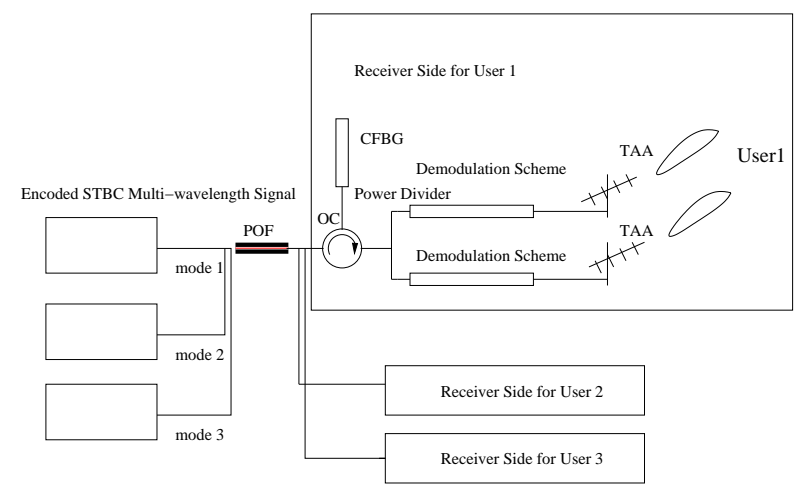

Figure 3: A Multi-user System

offering beamforming for the same signal after photo-detection and demodulation.

As demonstrated in Figure 2, after the CFBGs, the delayed wavelengths will be power-divided into two different demodulation schemes (DS), where each one contains several branches. The number of branches depends on the number of antenna elements. As shown in the bottom box of Figure 2, in each branch of DS 2, the multi-wavelength signal is split by a OBPF to retain half of the optical carrier having the corresponding wavelength and the right side-band which is modulated by $x_{2}$. By contrast, in DS 1 , the left side-band is filtered out. Thus, during the demodulation, the delayed photodetected signal is categorized into one of two groups and it is mapped to two antennas according to the filtered-out sidebands. For each antenna, we arrange for a linearly increasing time delay after the photo-detection of each wavelength, which is then exploited for beamsteering [2]. The desired beamsteering direction is determined by using explicit beamforming feedback, where a beamforming weight matrix containing the phase information for each antenna element is sent back from the desired users to the TAAs [19]. Then, in our design, this information is fed back over the fiber to the tunable frequency generator of Figure 2 through the POF, where the corresponding driving frequencies are applied. Explicitly, the AoD can be accurately estimated using classic techniques reported in the literature, such as the direction search for the largest gain used in the IEEE 802.11ad standard [14], the Kalman filter based tracking algorithm presented in [14] and the path search techniques using different beamwidths [15]. Additionally, several channel estimation technniques have been proposed for STBC in the literature, including closed-form blind channel estimation [16]. Then, the AoD information can be sent back to the tunable frequency generator to adjust the corresponding beamsteering angles.

Consequently, the specific pair of data streams modulated using STBC and carried by the same Radio Frequency (RF) 
will be beamsteered. Additionally, to appropriately tune the beamsteering direction, a tunable microwave generator used as the drive signal is imposed on the MZM2 for the sake of controlling the spacing of each wavelength, hence indirectly resulting in an adjustable beamforming pattern. The multiwavelength generation, tunable microwave generator and the multi-user system design will be described in the following sections.

\section{A. MZM-Aided Multi-wavelength Generation}

As shown in Figure 2, the multi-wavelength signal is generated with the aid of the MZM's nonlinearity, where the ODSB appears at every wavelength [17]. This is arranged for ensuring that different wavelengths experience different delays in the CFBG and hence beamforming can be achieved for each STBC signal, which is a benefit of the CFBG-inducedtime-delay of each wavelength. Here, we briefly introduce the physical rationale for this process.

The RF signal is directly modulated by laser, which can be formulated as:

$$
E_{d m}=\sqrt{P_{l a s e r}} e^{j 2 \pi f_{o c} t}\left[1+\cos \left(2 \pi f_{R F} t\right)\right],
$$

where $E_{d m}$ is the output field of the direct-modulated LD that operates at an optical power of $P_{\text {laser }}$ and an optical frequency of $f_{o c}$, which is modulated by a RF signal of frequency $f_{R F}$.

As detailed in [20], higher-order optical side-bands can be generated based on the voltage of the drive signal imposed on the MZM, which results in the optical signal as [17]:

$$
\begin{gathered}
E_{M Z M}=\cos \left( \pm \frac{\pi}{4}+\frac{\pi V_{d r} \cos \left(\omega_{L O}\right)}{2 V_{\pi}}\right) E_{d m}(t) \\
=\frac{E_{d m}(t)}{\sqrt{2}}\left[J_{0}\left(\frac{\pi\left|V_{d r}\right|}{2 V_{\pi}}\right)+2 \sum_{n=1}^{\infty}(-1)^{n} J_{2 n}\left(\frac{\pi V_{d r}}{2 V_{\pi}}\right) \cos \left(2 n \omega_{L O} t\right)\right. \\
\left. \pm 2 \sum_{n=1}^{\infty}(-1)^{n} J_{2 n-1}\left(\frac{\pi V_{d r}}{2 V_{\pi}}\right) \cos \left((2 n-1) \omega_{L O} t\right)\right],
\end{gathered}
$$

where the $E_{M Z M}$ is the output field of MZM2 seen in Figure 2 having a switching voltage of $V_{\pi}$, which imposes a phase-shift of $\pi$ for each arm of the MZM, while $V_{d r}$ is the drive voltage having the center angular frequency of $w_{L O} /(2 \pi)$. Finally, $J_{n}\left(\frac{\pi V_{d r}}{2 V_{\pi}}\right)$ is the Bessel function of the first kind and order $n$, which determines the number of side-bands.

The multi-wavelength signal generated is attributed to the changing of side-bands, which rely on $V_{d r}$. Thus, copies of ODSB can be readily obtained by simply tuning the voltage of the drive signal. However, to achieve convenient beamsteering tunability, in the following section a novel tunable microwave generator capable of generating the drive signal of MZM2 seen in Figure 2 is described.

\section{B. Tunable Frequency Generator}

In our system, CFBG is used for adjusting the time-delay imposed on the optical carriers, which can then be exploited for beamsteering [21]. This requires multiple wavelengths carrying the same signal and hence we utilize a MZM to create the multi-wavelength signal mentioned in Section II-A. However, for tuning the beamsteering direction and meet the minimum wavelength spacing requirement of $12.5 \mathrm{GHz}$ to avoid inter-channel crosstalk in optical transmission [22], a tunable microwave generator is required for the MZM's drive signal. Thus, we created the low-complexity tunable frequency generator of Figure 2 relying on another MZM modulator. Similar to the analysis in Section II-A, the higher-order sidebands resulting from the MZM's nonlinearity combined with a voltage-controllable drive signal will create a drive-voltagedependent optical signal.

As depicted in Figure 2, a four-side-band signal is generated by MZM1, where the optically modulated output signal is filtered by a notch filter (optical band stop signal) to retain merely the leftmost and rightmost side-bands, denoted here by $f_{1}$ and $f_{4}$. Thus, after photo-detection, the frequency of the demodulated RF signal will be twice that of the left/rightmost side-band. In the example of Figure $2,\left(f_{4}-f_{1}\right)$ is the output used for the drive signal of MZM2, which depends on the drive signal of MZM1 and on the number of the side-bands generated. Explicitly, the edge side-bands rely on the amplitude variation of the drive voltage, which will then control the frequencies generated by photo-detection. A tunable lower frequency generator can be used for generating microwave frequency. Thus, compared to commercially available microwave generators, this is a more cost-efficient solution for providing tunable frequencies.

\section{MDM based multi-user design}

POF is referred to as a multi-mode fiber [1] and can be used for the currently fast developing fiber technique of MDM [23]. Mode division multiplexing is a technique coupling different signals into different modes of multi-mode fiber. Here, we use POF as a mode division multiple access channel. As shown in Figure 3, each user's data feeds one of the modes of POF and shares the same receiver design, while each user can tune its own angle independently. Thus, the beam pattern can be adjusted and avoid interference while supporting multiple users thanks to our system's flexibility of independent tuning processes for different users.

To verify our proposed system, we consider twin-antenna based STBC modulation and each of the two STBC antenna arrays is equipped with four beamforming elements, as seen in Figure 2 and detailed in the following section.

\section{Simulation Results}

Table I: Simulation Parameter

\begin{tabular}{|l|l|}
\hline Parameter & Value \\
\hline RF signal & $2.4 \mathrm{GHz}$ \\
\hline LD Center Wavelength & $1550 \mathrm{~nm}$ \\
\hline Drive Frequency (MZM) & $12.5 \mathrm{GHz}$ \\
\hline MZM mode & push-pull mode \\
\hline Fiber type & POF \\
\hline Fiber length & $40 \mathrm{~m}$ \\
\hline Channel Model & Power Flow Equation \\
\hline CFBG length & $22 \mathrm{~cm}$ \\
\hline Diversity employed & STBC \\
\hline Modulation Type & DBPSK \\
\hline Simulation Environment & MATLAB \\
\hline
\end{tabular}

In this section, we consider a system to support the WiFi spectrum of $2.4 \mathrm{GHz}$, where each user is supported by 
MF-MIMO transmission intrinsically combining twin-antenna STBC and beamforming. We consider beamforming using four antenna elements per antenna array. Table I shows the simulation parameters, where after STBC encoding [8], the Differential Binary Phase Shift Keying (DBPSK) STBC signal is direct-modulated by two separate LDs. The MZM is based on the Quadrature Point and operates in the push-pull mode [17]. Furthermore, $\frac{\pi V_{d r}}{2 V_{\pi}}$ is set to 0.13 , where the third side band can be ignored, due to the low power level, as shown in Figure 4.

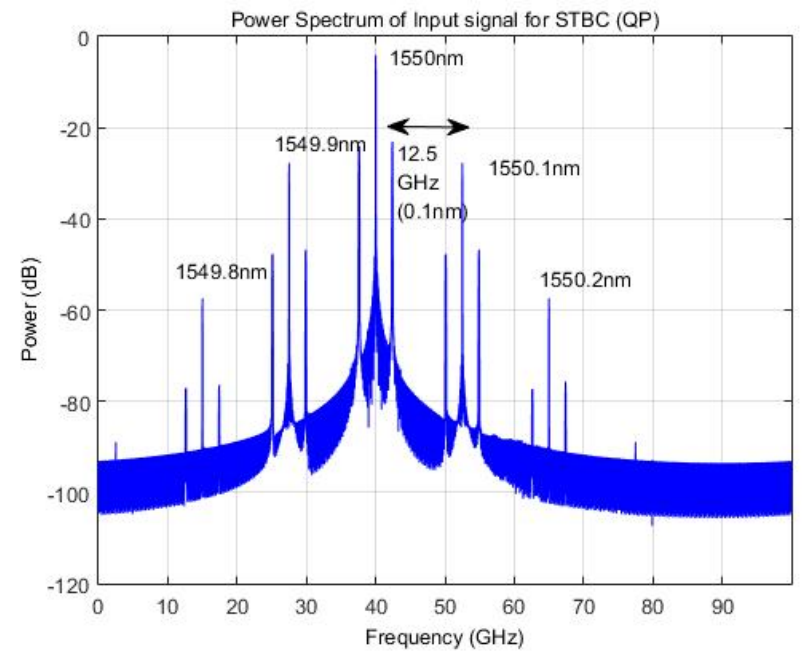

Figure 4: MZM aided multi-wavelength generation

In Figure 4, we opted for $40 \mathrm{GHz}(1550 \mathrm{~nm})$ as the center frequency for the simulations and a wavelength spacing of 12.5 $\mathrm{GHz}$ is used. It is shown in Figure 4 that the same pair of STBC signal is modulated to the ODSBs carried by different wavelengths. A short plastic optical fiber of $40 \mathrm{~m}$ is simulated based on Mateo's power flow equation detailed in [18], which is suitable for mode division multiplexing, where the multi-wavelength signal is coupled into one of the mode in the POF. A $22 \mathrm{~cm}$ CFBG is used for introducing the delay required at the different wavelengths in order to impose the specific time delay required for beamsteering. The time delay imposed on the different wavelengths is simulated by using the OPTIGRATING software [24] as shown in Figure 5, where the delay is a linear function of the wavelengths ranging from 1549 to $1551 \mathrm{~nm}$.

Arranging for a constant wavelength-spacing guarantees the required equal time delay difference of neighbouring wavelengths due to the linear time delay of the CFBG, as shown in Figure 5. This introduces a linearly increasing time delay between the adjacent elements of each antenna, hence resulting in beamsteering in the wireless transmitter. Here, according to the gradient of the linear part shown in Figure 5 , we can readily obtain the time delay for the wavelength spacing of $0.1 \mathrm{~nm}(12.5 \mathrm{GHz})$ as $78 \mathrm{ps}$.

To control the beamsteering direction, the tunable range of frequencies in Figure 2 spans from $12.5 \mathrm{GHz}$ to $31.5 \mathrm{GHz}$ with a step size of $1 \mathrm{GHz}$. This correponds to a time delay difference ranging from of $78 \mathrm{ps}$ to $200 \mathrm{ps}$, with a step size of approximately 6 ps. This allows us to tune the beamforming pattern of a four-element antenna, as shown in Figure 6, where the beamforming pattern of each step changes from the right

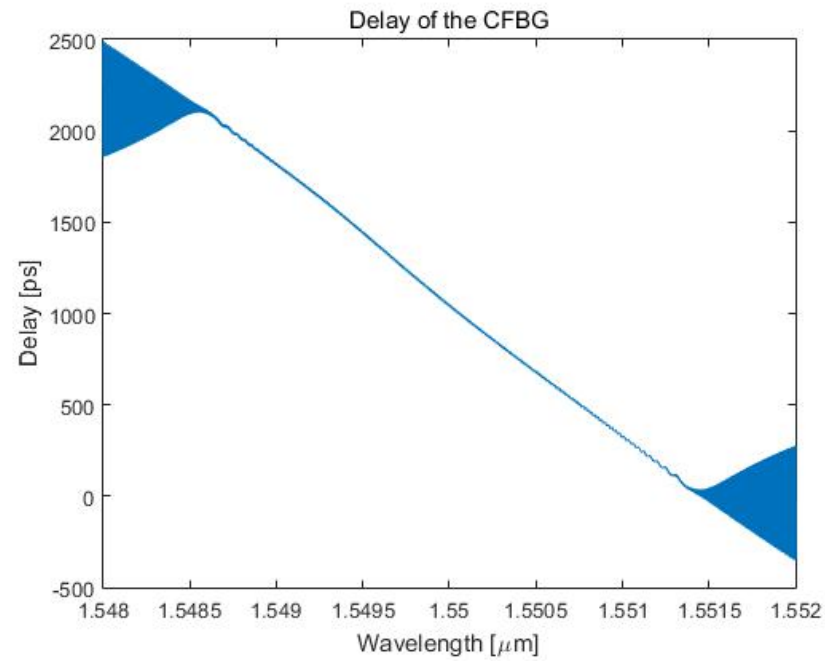

Figure 5: Time Delay of CFBG

to left in a very small step. It is clearly shown in Figure 6 that the beamsteering angular range is upto $150^{\circ}$, which is suitable for indoor transmissions.

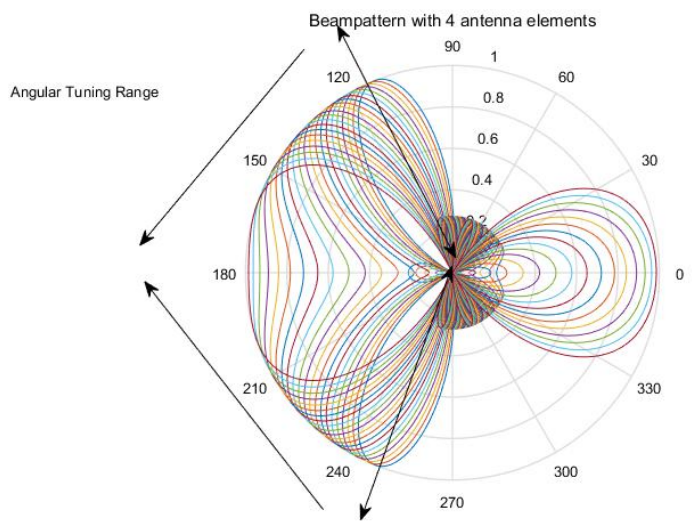

Figure 6: Beamforming Pattern

The system is scalable, where by carefully tuning the drive signal voltage of MZM2, the number of wavelengths will be changing, adapting itself to different antenna sizes. For example, more than two side-bands can be obtained by the MZM-Aided multi-wavelength generation mentioned in Section II, narrowing the beams and thus increasing the propagation range.

Additionally, MDM introduced by POF is capable of supporting multiple user transmissions, as shown in Figure 3, where user 1,2 and 3 share the same architecture before the POF of Figure 2. User 1, 2 and 3 encode different sets of STBC signals with the aid of the same architecture and these signals are transmitted in different modes of the POF through MDM. At the receiver side of POF, the demodulation is the same as that of the single-user system of Figure 2. In order to show how the different users' signals can be tuned using the proposed architecture, Figures 7 and 8 show different beams for three users. In the example of Figures 7 and 8, three users are located at angles of $110^{\circ}, 70^{\circ}$ and $140^{\circ}$, which correspond to time delay differences of $78 \mathrm{ps},-78 \mathrm{ps}$ and $162 \mathrm{ps}$. Figure 7 


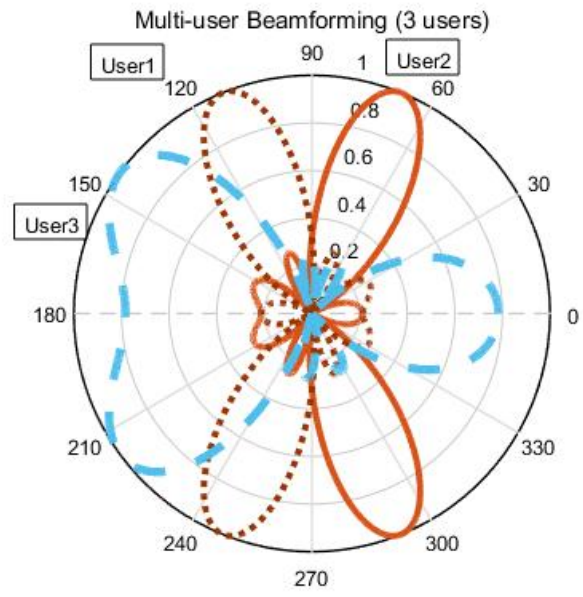

Figure 7: A Multi-user System Beampattern ( $\mathrm{N}=4$ antenna elements)

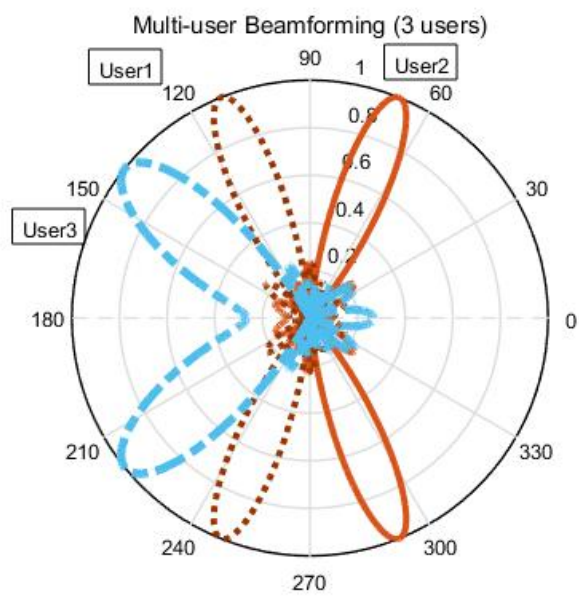

Figure 8: A Multi-user System Beampattern ( $N=7$ antenna elements)

shows the beams, when $\mathrm{N}=4$ antenna elements are used, while Figure 8 shows the beams, when $\mathrm{N}=7$ antenna elements are employed.

\section{CONCLUSIONS}

A novel all-optical processing aided multi-functional wireless MIMO system was designed by exploiting the MZM's nonlinearity and CFBG, where a POF was used for supporting a multi-user system. Furthermore, a novel tunable microwave generator was conceived for feeding the MZM drive signal as a more cost-efficient solution than its commercial counterpart. The ODBS-encoded STBC signal was realized by using a combination of two LDs and two BPFs, where the associated multi-wavelength generation is based on the MZM's nonlinearity. Our design achieved a beamforming angular range of upto $150^{\circ}$ with a small time delay step of $6 \mathrm{ps}$, which is suitable for indoor communications.

\section{REFERENCES}

[1] A. Koonen and E. Tangdiongga, "Photonic Home Area Networks," Journal of Lightwave Technology, vol. 32, no. 4, pp. 591-604, 2014
[2] Z. Cao, Q. Ma, A. B. Smolders, Y. Jiao, M. J. Wale, C. W. Oh, H. Wu, and A. M. J. Koonen, "Advanced Integration Techniques on Broadband Millimeter-Wave Beam Steering for 5G Wireless Networks and Beyond," IEEE Journal of Quantum Electronics, vol. 52, no. 1, pp. 1-20, 2016.

[3] M. El-Hajjar and L. Hanzo, "Multifunctional MIMO systems: A Combined Diversity and Multiplexing Design Perspective," IEEE Wireless Communications, vol. 17, pp. 73-79, April 2010.

[4] L. Hanzo, M. El-Hajjar, and O. Alamri, "Near-Capacity Wireless Transceivers and Cooperative Communications in the MIMO Era: Evolution of Standards, Waveform Design, and Future Perspectives," Proceedings of the IEEE, vol. 99, pp. 1343-1385, Aug 2011.

[5] J. Kim and I. Lee, "802.11 WLAN: history and new enabling MIMO techniques for next generation standards," IEEE Communications Magazine, vol. 53, pp. 134-140, March 2015.

[6] Y. Shi, M. Morant, C. Okonkwo, R. Llorente, E. Tangdiongga, and A. Koonen, "Multistandard Wireless Transmission Over SSMF and Large-Core POF for Access and In-Home Networks," IEEE Photonics Technology Letters, vol. 24, pp. 736-738, May 2012

[7] G. Jongren, M. Skoglund, and B. Ottersten, "Combining beamforming and orthogonal space-time block coding," IEEE Transactions on Information Theory, vol. 48, pp. 611-627, Mar 2002.

[8] A. Goldsmith, Wireless communications. Cambridge university press, 2005.

[9] I. S. Amiri, S. E. Alavi, N. Fisal, A. S. M. Supa'at, and H. Ahmad, "AllOptical Generation of Two IEEE802.11n Signals for $2 \times 2$ MIMO-RoF via MRR System," IEEE Photonics Journal, vol. 6, pp. 1-11, Dec 2014

[10] V. Italia, M. Pisco, S. Campopiano, A. Cusano, and A. Cutolo, "Chirped fiber Bragg gratings for electrically tunable time delay lines," IEEE Journal of Selected Topics in Quantum Electronics, vol. 11, pp. 408-416, March 2005

[11] V. A. Thomas, M. El-Hajjar, and L. Hanzo, "Single ODSB Radio-OverFiber Signal Supports STBC at Each RAP," IEEE Communications Letters, vol. 19, pp. 1331-1334, Aug 2015.

[12] I. A. Hemadeh, M. El-Hajjar, S. Won, and L. Hanzo, "Multi-set spacetime shift-keying with reduced detection complexity," IEEE Access, vol. 4, pp. 4234-4246, 2016.

[13] B. Friedlander and S. Scherzer, "Beamforming versus transmit diversity in the downlink of a cellular communications system," IEEE Transactions on Vehicular Technology, vol. 53, pp. 1023-1034, July 2004.

[14] C. Zhang, D. Guo, and P. Fan, "Tracking angles of departure and arrival in a mobile millimeter wave channel," in 2016 IEEE International Conference on Communications (ICC), pp. 1-6, May 2016.

[15] A. Alkhateeb, O. E. Ayach, G. Leus, and R. W. Heath, "Channel estimation and hybrid precoding for millimeter wave cellular systems," IEEE Journal of Selected Topics in Signal Processing, vol. 8, pp. 831846 , Oct 2014

[16] S. Shahbazpanahi, A. B. Gershman, and J. H. Manton, "Closed-form blind MIMO channel estimation for orthogonal space-time block codes," IEEE Transactions on Signal Processing, vol. 53, pp. 4506-4517, Dec 2005.

[17] V. A. Thomas, M. El-Hajjar, and L. Hanzo, "Millimeter-Wave Radio Over Fiber Optical Upconversion Techniques Relying on Link Nonlinearity," IEEE Communications Surveys Tutorials, vol. 18, pp. 29-53, Firstquarter 2016.

[18] J. Mateo, M. A. Losada, I. Garcés, and J. Zubia, "Global characterization of optical power propagation in step-index plastic optical fibers.," Optics express, vol. 14, no. 20, pp. 9028-9035, 2006.

[19] "IEEE Standard for Information technology-Telecommunications and information exchange between systems Local and metropolitan area networks-Specific requirements - Part 11: Wireless LAN Medium Access Control (MAC) and Physical Layer (PHY) Specifications," IEEE Std 802.11-2016 (Revision of IEEE Std 802.11-2012), pp. 1-3534, Dec 2016.

[20] S. Ghafoor and L. Hanzo, "Reduced Dispersion Duplex DQPSK RadioOver-Fiber Communications Using Single-Laser-Based Multiple SideBands," in 2011 IEEE International Conference on Communications (ICC), pp. 1-5, June 2011.

[21] C. A. Balanis, Antenna theory: analysis and design, vol. 1. John Wiley \& Sons, 2005.

[22] G. P. Agrawal, Fiber-optic communication systems, vol. 222. John Wiley \& Sons, 2012

[23] D. J. Richardson, J. M. Fini, and L. E. Nelson, "Space-division multiplexing in optical fibres," Nat Photon, vol. 7, pp. 354-362, May 2013. Review.

[24] "Fiber Bragg Grating", Optiwave,2017.[Online].Available: https://optiwave.com/resources/applications-resources/optical-gratingfiber-bragg-grating/. [Accessed: 14-March-2017]. 